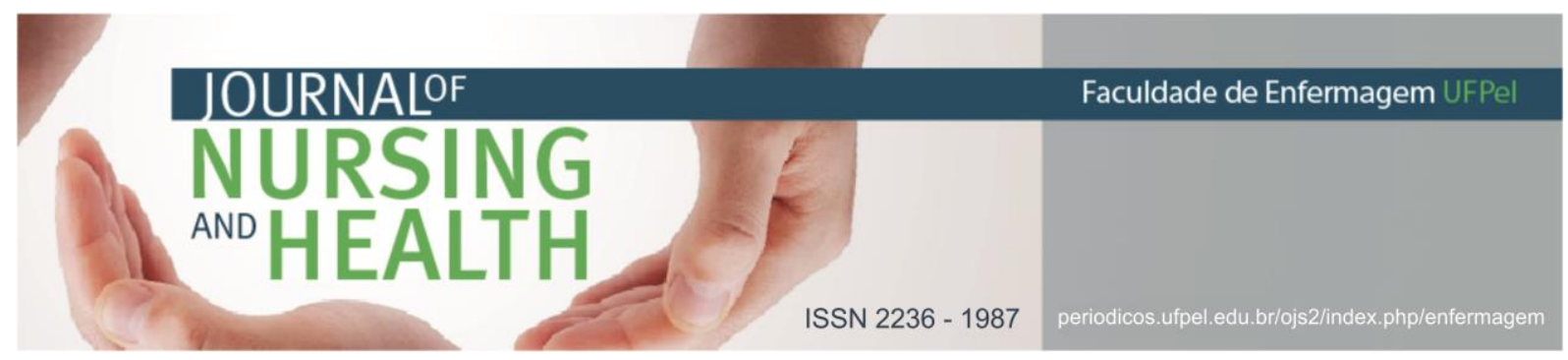

ARTIGO DE REVISÃO

\title{
Sexualidade e saúde mental: uma revisão integrativa
}

\author{
Sexuality and mental health: an integrative review
}

\section{La sexualidad y la salud mental: una revisión integradora}

Maidana Júnior, João Nunes ${ }^{1}$ Viana, Diogo da Rosa²; Siniak, Débora Schlotefeldt³; Lipinski, Jussara Mendes ${ }^{4}$

Como citar este artigo: Maidana Júnior JN, Viana DR, Siniak DS, Lipinski JM. Sexualidade e saúde mental: uma revisão integrativa. J nurs health. 2018;8(3):e188304

\section{RESUMO}

Objetivo: conhecer a produção científica brasileira acerca da sexualidade de pessoas com transtornos mentais. Métodos: revisão integrativa realizada através de uma busca das produções científicas dos últimos dez anos na Biblioteca Virtual em Saúde, no período de janeiro a março de 2016. Foram obtidos 1.721 artigos e após a seleção foram incluídos seis estudos. Resultados: agrupou-se os resultados em duas principais categorias. Com relação à visão dos profissionais foi observada a fragilidade das equipes de saúde para lidarem com questões relacionadas à sexualidade das pessoas com transtorno mental. Além disso, notou-se a presença de preconceitos, tabus e a negação da existência da sexualidade não patológica nestas pessoas. Considerações Finais: considera-se imprescindível a sensibilização dos profissionais, para que as pessoas com transtornos mentais assumam o protagonismo da própria vida e vivam sua sexualidade.

Descritores: Sexualidade; Saúde mental; Cuidados de enfermagem.

\begin{abstract}
Objective: to know Brazilian scientific production about the sexuality of people with mental disorders. Methods: integrative review carried out through a search of the scientific productions of the last ten years in the Virtual Health Library, from January to March, 2016. There were obtained 1,721 articles and six studies were included after the selection. Results: the results were grouped into two main categories. Regarding the professionals' view, the fragility of the health teams to deal with issues related to the sexuality of people with mental disorders was observed. In addition, it was noted the presence of prejudices, taboos and the denial of non-pathological sexuality in these people. Final Considerations: it is considered essential to raise the awareness of professionals, so that people with mental disorders are protagonists of their lives and their sexuality.

Descriptors: Sexuality; Mental health; Nursing care.
\end{abstract}

\footnotetext{
1 Discente da Graduação de Enfermagem. Universidade Federal do Pampa. E-mail: juniordana@hotmail.com http://orcid.org/0000-0001-8411-5566

2 Discente da Graduação de Enfermagem. Universidade Federal do Pampa. E-mail: diogoviana95@yahoo.com.br http://orcid.org/0000-0001-6704-8103

3 Enfermeira. Mestre em Enfermagem. Universidade Federal do Pampa. E-mail: debynha33@hotmail.com http://orcid.org/0000-0002-7689-6953

4 Enfermeira. Doutora em Enfermagem. Universidade Federal do Pampa. E-mail: jussaralipinski@gmail.com. http: / / orcid.org/0000-0002-3907-0722
} 


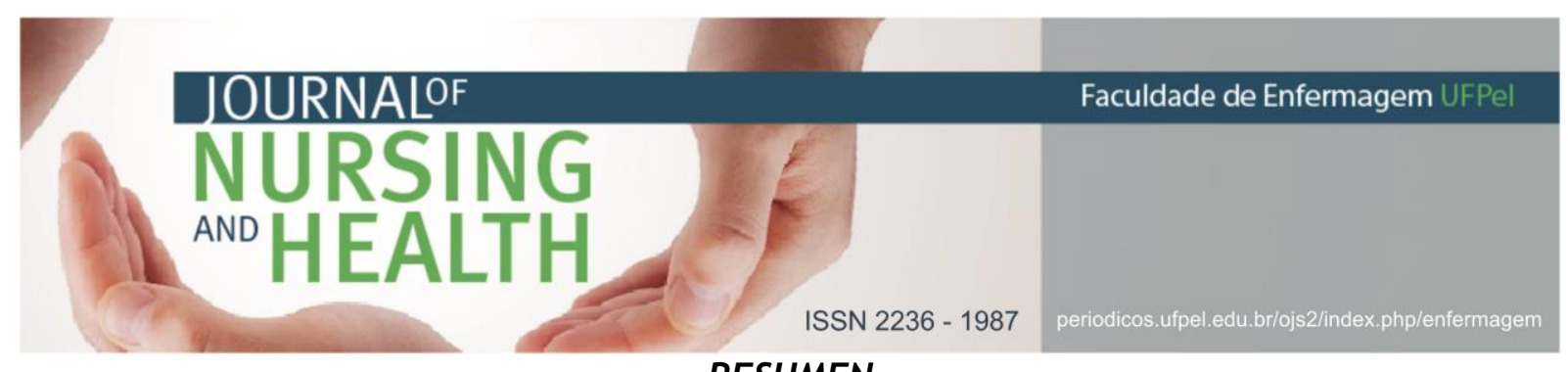

RESUMEN

Objetivo: conocer la producción científica brasileña acerca de la sexualidad de las personas con trastornos mentales. Métodos: una revisión integradora llevado a cabo a través de una búsqueda de la producción científica de los últimos diez años en la Biblioteca Virtual en Salud, en el período enero-marzo de 2016 se obtuvieron 1.721 artículos y después de la selección se incluyeron seis estudios. Resultados: se agruparon los resultados en dos categorías principales. En cuanto a la visión profesional se observó la fragilidad de los equipos de salud para tratar los temas relacionados con la sexualidad de las personas con trastorno mental, además, destacó la presencia de prejuicios, tabúes y la negación de la existencia de la sexualidad no patológico en estas personas. Consideraciones finales: se considera esencial para sensibilizar a los profesionales, por lo que las personas con trastornos mentales sean protagonistas de sus vidas y su sexualidad.

Descriptores: Sexualidad; Salud mental; Atención de enfermería.

\section{INTRODUÇÃO}

0 processo da reforma psiquiátrica surgiu como uma mudança de paradigma na esfera do cuidado às pessoas com sofrimento psíquico. Ocorreram transformações em diversos aspectos, incluindo as dimensões epistemológica, jurídico-política, técnica e cultural buscando a desconstrução do aparato manicomial. 1

Dentre os desafios a serem superados nesse novo modelo de atenção, destaca-se a busca de um cuidado ampliado aos usuários e que considere, as questões complexas que os permeiam, como a sexualidade desses indivíduos. Desta forma, diferente do modelo manicomial, as práticas psicossociais precisam ser promovidas a partir da vida singular e contextual do sujeito que sofre. ${ }^{2}$

A saúde sexual é a habilidade de mulheres e homens de desfrutar e expressar sua sexualidade, sem riscos de infecções sexualmente transmissíveis, gestações não desejadas, coerção, violência e discriminação. É possível experimentar uma vida informada, agradável e segura, baseada na autoestima, que implica abordagem positiva e respeito mútuo nas relações sexuais. ${ }^{3}$

Sendo assim, ela está relacionada diretamente aos direitos reprodutivos, de desejar ou não ter filhos, escolha do momento certo, direitos de informações quanto aos métodos contraceptivos e de exercerem a sexualidade livre de discriminação. ${ }^{3}$ Neste sentido, entende-se que este conceito está atrelado à perspectiva de clínica ampliada, onde se compreende o sujeito para além da doença e que ocupa diferentes espaços e papéis na sociedade.

Neste sentido, este estudo tem como objetivo conhecer a produção científica brasileira acerca da sexualidade de pessoas com transtornos mentais.

\section{MATERIAIS E MÉTODOS}

Este estudo é uma revisão integrativa que consiste em método de reunião e síntese de resultados de investigações, originalmente construído a partir das áreas de educação e psicologia. ${ }^{4}$ Esta revisão seguiu cinco etapas: a) formulação da questão norteadora; b) coleta de 


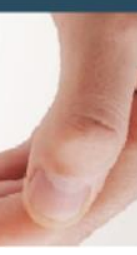

ISSN 2236 - 1987

dados; c) avaliação dos dados coletados; d) análise e interpretação dos dados; e) apresentação dos resultados. ${ }^{4}$

Inicialmente formulou-se a seguinte questão norteadora: qual produção científica brasileira sobre a temática de sexualidade de mulheres com transtorno mental?

A partir da definição da questão de pesquisa iniciou-se a busca das produções científicas por meio da Biblioteca Virtual da Saúde (BVS). A busca foi realizada no período de janeiro a março de 2016, utilizando-se os seguintes descritores: "sexualidade" and "saúde mental".

Para inclusão dos estudos utilizou-se os seguintes critérios: artigos, que contemplassem a temática de sexualidade de mulheres com transtorno mental, estar disponível na forma completa e gratuita em português, publicados entre 2005 e 2015, justifica-se tal recorte, uma vez que, em pesquisa prévia acerca das produções dos últimos cinco anos o quantitativo de trabalhos que abordavam a temática foi inexpressivo. Foram excluídas dissertações e teses.

Com base nestes descritores foram identificadas 1.721 produções. A seguir foi realizado um refinamento com o uso dos filtros de acordo com os critérios de inclusão, o que resultou em 141 artigos, os demais 1.580 embora tivessem os descritores não se adequavam a temática proposta.

Após esta etapa realizou-se a leitura de títulos e resumos dos estudos a fim de verificar se contemplavam de fato a temática proposta, sendo assim, compuseram o corpus do estudo seis artigos identificados com a letra $\mathrm{A}$ de artigo e números arábicos de 1 a 6, sendo A1, A2 e assim sucessivamente, dos quais realizou-se leitura na íntegra, possibilitando a obtenção dos dados que subsidiaram a discussão.

\section{RESULTADOS E DISCUSSÃO}

Ao analisar os estudos selecionados, observou-se quanto ao ano de publicação que em 2013 foram publicados dois artigos $(33,4 \%)$, seguido de um artigo $(16,7 \%)$ em 2008 , 2009 e 2010.

O centro de pesquisa de maior produção foi a Universidade Federal de Minas Gerais, com duas publicações $(33,4 \%)$. Com uma produção cada $(16,7 \%)$ aparecem a Universidade Regional de Cariri, Universidade Federal de São Paulo, Universidade Nove de Julho em parceria com a Universidade Federal de São Paulo e Universidade Federal do Rio Grande do Norte em parceria com a Universidade de São Paulo.

Em relação às regiões do Brasil em que os estudos foram realizados, cinco são oriundos da região Sudeste $(83,5)$ e um da região Nordeste $(16,7 \%)$. Sendo que as pesquisas apresentam abordagem qualitativa de forma em $100 \%$ dos artigos. Os resultados estão apresentados na Tabela 1.

Da leitura minuciosa dos seis artigos elencados para estudo, algumas temáticas emergiram de forma mais intensa e sobre as quais colocaremos nosso olhar baseado nas vivências pessoais, acadêmicas e profissionais. Nestes estudos foi possível observar que cinco deles trazem a visão do 
profissional em relação à sexualidade das pessoas com transtornos mentais, que deu origem a duas categorias: Sexualidade como manifestação de doença e o cuidado em saúde mental: a visão dos profissionais e Sexualidade negada: Preconceitos, tabus, vigilância e punição

Tabela 1 - Resultado da pesquisa nas bases de dados e estudos selecionados.

\begin{tabular}{|c|c|c|c|c|c|}
\hline $\begin{array}{l}\text { Código } \\
\text { do } \\
\text { Artigo }\end{array}$ & $\begin{array}{c}\text { Base } \\
\text { de } \\
\text { dados }\end{array}$ & Título & Autor & $\begin{array}{c}\text { Ano de } \\
\text { Publicação }\end{array}$ & $\begin{array}{l}\text { Local } \\
\text { de } \\
\text { estudo }\end{array}$ \\
\hline $\mathrm{A} 1^{5}$ & BVS & $\begin{array}{l}\text { A sexualidade negada do } \\
\text { doente mental: percepções } \\
\text { da sexualidade do portador } \\
\text { de doença mental por } \\
\text { profissionais de saúde }\end{array}$ & $\begin{array}{c}\text { Patrícia Franscisca de } \\
\text { Brito; } \\
\text { Cleide Correia de } \\
\text { Oliveira }\end{array}$ & 2009 & CE \\
\hline$A 2^{6}$ & BVS & $\begin{array}{c}\text { Percepções de } \\
\text { trabalhadores de } \\
\text { enfermagem sobre a } \\
\text { sexualidade de portadores } \\
\text { de transtornos mentais }\end{array}$ & $\begin{array}{l}\text { Gisela Cardoso } \\
\text { Ziliotto; João } \\
\text { Fernando Marcolan }\end{array}$ & 2013 & SP \\
\hline $\mathrm{A} 3^{7}$ & BVS & $\begin{array}{l}\text { Representações sociais de } \\
\text { enfermagem: a sexualidade } \\
\text { de portadores de } \\
\text { transtornos mentais }\end{array}$ & $\begin{array}{l}\text { Gisela Cardoso } \\
\text { Ziliotto; João } \\
\text { Fernando Marcolan }\end{array}$ & 2014 & SP \\
\hline $\mathrm{A} 4^{8}$ & BVS & $\begin{array}{l}\text { Práticas discursivas e o } \\
\text { silenciamento do doente } \\
\text { mental: sexualidade } \\
\text { negada? }\end{array}$ & $\begin{array}{l}\text { Francisco Arnoldo } \\
\text { Nunes de Miranda; } \\
\text { Antonia Regina } \\
\text { Ferreira Furegato; } \\
\text { Dulcian Medeiros de } \\
\text { Azevedo }\end{array}$ & 2008 & SP \\
\hline$A 5^{9}$ & BVS & $\begin{array}{l}\text { Oficina de sexualidade em } \\
\text { saúde mental: relato de } \\
\text { experiência }\end{array}$ & $\begin{array}{c}\text { Marlene Alves da Silva } \\
\text { e Cláudia Garcia } \\
\text { Capitão }\end{array}$ & 2010 & MG \\
\hline$A 6^{10}$ & BVS & $\begin{array}{c}\text { Sexualidade e } \\
\text { vulnerabilidade social em } \\
\text { face das infecções } \\
\text { sexualmente transmissíveis } \\
\text { em pessoas com transtorno } \\
\text { mental }\end{array}$ & $\begin{array}{l}\text { Jaqueline Almeida } \\
\text { Guimarães Barbosa; } \\
\text { Mark Drew Crosland } \\
\text { Guimarães; Maria } \\
\text { Imaculada de Fátima } \\
\text { Freitas } \\
\end{array}$ & 2013 & $M G$ e $R J$ \\
\hline
\end{tabular}

Sexualidade como manifestação de doença e o cuidado em saúde mental: a visão dos profissionais

0 artigo $A 1,{ }^{5}$ ressalta que a ótica dos profissionais se refere somente ao ato sexual em si. A verbalização intensa do desejo de praticar tal ação manifestada pelo doente, para a maioria dos deles é vista como um estado delirante (surto). ${ }^{5}$ A leitura permite observar que eles encaram a essas manifestações como algo exacerbado e descontrolado como o próprio doente. 0 estudo aponta que a forma como as enxergam é carregada pela repressão e negação de tais manifestações. 
Ainda com relação à visão das fragilidades apresentada pelas equipes para lidarem com questões relacionadas à sexualidade dessas pessoas, onde aparece a negação da existência de uma sexualidade não patológica nestas pessoas, pois ela é parte integrante e inerente aos seres humanos, necessitando ser refletida e debatida por todos.

No estudo $A 2,{ }^{6}$ realizado com 07 enfermeiras e 11 auxiliares de enfermagem, os autores concluíram que a conduta de enfermagem frente à sexualidade das pessoas com transtorno mental incorporou um dispositivo de disciplina, vigilância, controle e punição, alertando também sobre a falta de capacitação para os profissionais de enfermagem frente à temática, ressalta ainda, que os profissionais, agem como se o portador de doença mental fosse um ser assexuado. ${ }^{6}$

A experiência tem demonstrado que quando as mulheres com transtornos mentais manifestam seus desejos estes tendem a ser condenados e reprimidos, já em relação aos homens a percepção é diferente, muitas vezes buscando compreender e ou justificar suas ações e reações.

Assim, percebe-se a necessidade da desconstrução de preconceitos sociais e culturais, associados a uma visão conservadora na qual alguns profissionais compreendem a sexualidade como inerente apenas ao homem, atitude que acaba por reproduzir uma conjuntura histórica de repressão às mulheres.
Reforçando com os demais estudos, $A 3^{7}$ concluiu que o enfermeiro e os demais profissionais de saúde negam a sexualidade das pessoas com transtornos mentais. As pesquisadoras ressaltam que essa incapacidade dos profissionais de entenderem a sexualidade do portador de transtorno mental possui raiz na sua formação, quando não é abordada essa temática. Por fim, concluíram que as crenças, juízos de valores e estigma dos trabalhadores da enfermagem, interferem de forma negativa na assistência prestada. ${ }^{7}$ Estes resultados também reforçam os achados emergidos do artigo A2. ${ }^{6}$

Em pesquisa realizada com 17 enfermeiros que trabalham em serviços psiquiátricos $A 4,{ }^{8}$ verificou que existe de um lado o ocultamento dessa temática no meio profissional e de outro existe a opressão e dispersão da informação sobre a sexualidade. ${ }^{8}$

Neste sentido, compreende-se que ao impor suas percepções, o profissional passa a exercer controle sobre seus corpos e ações, negando ao ser humano o direito de viver explorar seu corpo e sua sexualidade conforme lhe apraz, cabendo aos profissionais servir como intermediário, entre a vivência da sexualidade e a difusão de informação.

0 artigo $A 5,{ }^{9}$ traz a interlocução entre as oficinas terapêuticas e educação em saúde, onde aparece que a sexualidade em pacientes com transtorno mental se manifesta, porém ainda é reprimida pelos profissionais. As descrições das oficinas revelam a participação ativa dos usuários, evidenciando demandas de momentos 


\section{ISSN 2236 - 1987}

de discussão e escuta sobre as questões de sexualidade. ${ }^{12}$ Pensar acerca deste fato, remete a importância de que espaços de trocas sejam estimulados, no qual as pessoas possam se manifestar com tranquilidade, sem se sentirem julgadas.

No estudo $A 2,6$ identificou-se que as manifestações mais singelas e rotineiras de expressão da sexualidade como toque, beijo, abraço, olhar ou modo de vestir-se também foram consideradas como fora do contexto esperado e, portanto, vistas como psicopatológicas. ${ }^{6}$

O estudo $A 3^{7}$ corrobora com os achados do $\mathrm{A} 2,{ }^{6}$ quando descreve que a maioria dos trabalhadores de enfermagem percebe a sexualidade do portador de transtorno mental como manifestação da doença, sendo o indivíduo incapacitado de expressão da sexualidade tida como normal, uma vez que não consegue regular seus afetos e desejos. $^{7}$

Como os demais artigos, $\mathrm{A} 4^{8}$ ainda reflete que os silenciamentos são articulados na trama diária do enfermeiro, requerendo um constante reequilíbrio diante das respostas que se situam entre a ciência e o senso comum sobre a sexualidade do portador de transtorno mental. ${ }^{8}$

Há alguns tipos de transtornos nos quais pode haver momentos de hipersexualidade, porém, tais casos, não são tão comuns podendo ocorrer em situações nas quais não há controle do quadro pelas terapias ou uso de medicação, neste sentido não devem ser realizadas generalizações.
Sexualidade negada: preconceitos, tabus, vigilância e punição

0 artigo $A 3^{7}$ reconheceu o quanto preconceito, crenças, juízos de valor e estigma de trabalhadores da enfermagem interferem de forma negativa na qualidade da assistência prestada. $^{7}$ Já A6 alerta ainda os profissionais de saúde, que a grande especificidade das pessoas com transtornos mentais é seu contexto social, neste caso marcado por pobreza, ignorância, abandono, preconceito e drogadição. Para os autores o contexto além de dificultar o autocuidado, se mostrou favorecedor de situações de mais vulnerabilidade, como de venda de sexo e violência sexual. ${ }^{11}$

Ressalta-se a importância dos profissionais de saúde, ampliarem seu olhar e seu fazer, na identificação de situações de vulnerabilidade, seja em questões de violência, prostituição e uso de drogas. Por isso, conhecer o contexto na qual essas pessoas estão inseridas, é fundamental para que o profissional seja capaz de planejar e implementar o cuidado de forma ampla.

Já o artigo $A 4,{ }^{8}$ relata que o enfermeiro desmente a evidência da sexualidade nessas pessoas, tornando toda forma de manifestação de sexualidade, como algo incompreensível. ${ }^{8}$

0 principal fator que impede uma abordagem permanente $\mathrm{da}$ sexualidade nos setores saúde e educação foi o despreparo profissional, tanto na formação inicial como na educação permanente. ${ }^{11}$ A negação da 


\section{JOURNAIOF

sexualidade pelos profissionais de saúde muitas vezes ocorre, pelos próprios tabus e preconceitos existentes com relação à própria sexualidade. Cabendo então a reflexão de quanto o agir, limite e cria lacunas na perspectiva da integralidade do cuidado.

Neste sentido, pensar em um espaço de desconstrução de estereótipos e problematização das noções, a partir de um olhar singular sobre os usuários, permite que estes também possam delinear estratégias de autocuidado. ${ }^{12}$ É necessário então, inserir essas discussões sobre sexualidade na graduação e na educação permanente como tema transversal. ${ }^{13}$

Há lacunas na assistência em saúde mental no que se refere à promoção da saúde e prevenção de doenças, um campo inerente à assistência de enfermagem é o que nos relata $45 .{ }^{9}$ Infelizmente, essas práticas são comuns dentro dos serviços substitutivos, porque se entende que existem fragilidades que precisam ainda ser supridas.

\section{CONSIDERAÇÕES FINAIS}

A realização desta revisão integrativa apresentou como maior limitação a baixa produção no Brasil acerca da sexualidade de pessoas com transtornos mentais.

Nos artigos revisados aparece de forma clara que a sexualidade destas pessoas ainda está atrelada a preconceitos, tabu e negligencias dos profissionais. Dos textos analisados, cinco abordaram a visão dos profissionais frente a sua compreensão acerca da sexualidade de pessoas com transtorno mental e apenas um artigo, apresentou a visão dos usuários, o que reforça que mesmo na pesquisa ainda se sobressai à exclusão das pessoas com transtorno mental, quando se trata de conhecer seus sentimentos, medos vivências e necessidades.

Nesse sentido, torna-se imprescindível a sensibilização dos diferentes profissionais, para que conheçam, respeitem e garantam o direito de homens e mulheres de assumirem o protagonismo da vida, lembrando que toda manifestação de sexualidade deve ser respeitada e cuidada para que possa ser vivenciada de forma segura e plena.

\section{REFERÊNCIAS}

1 Ferreira TPS, Sampaio J, Souza ACN, Oliveira DL, Gomes LB. Produção do cuidado em Saúde Mental: desafios para além dos muros institucionais. Interface comu saúde educ [Internet]. 2017 [acesso em 2018 set 20];21(61):373-84. Disponível em: http://www.scielo.br/scielo.php?scrip $\mathrm{t}=\mathrm{sci}$ arttext\&pid=S1414-

$32832017000200373 \& \operatorname{lng}=e n \& n r m=i s o$ \&tlng=pt

2 Grigolo TM, Peres GM, Junior CAG, Rodrigues J. $O$ projeto terapêutico singular na clínica da atenção psicossocial. Cadernos brasileiros de saúde mental [Internet]. 2015 jun/dez 73 [acesso em 2018 set 20];7(15):53-. Disponível em: http://incubadora.periodicos.ufsc.br/ index.php/cbsm/article/view/2951/4 437

3. Ministério da Saúde (BR). Secretaria de Atenção à Saúde. Departamento de 
Atenção Básica. Saúde sexual e saúde reprodutiva. Brasília; 2013.

4 Soares CB, Hoga LAK, Peduzzi M, Sangaleti C, Yonekura T, Silva DRAD. Revisão integrativa: conceitos e métodos utilizados na enfermagem. Rev esc enferm [Internet]. 2014 jan/jan [acesso em 2018 set 20];48(2):335-45. Disponível em: http://www.scielo.br/pdf/reeusp/v48 n2/pt_0080-6234-reeusp-48-02-

335.pdf

5 Brito PF, Oliveira CC. A sexualidade negada do doente mental: percepções da sexualidade do portador de doença mental por profissionais de saúde. Ciência e cognição [Internet]. 2009 nov/mar [acesso em 2018 set 20];14(1):246-54. Disponível em: http://pepsic.bvsalud.org/pdf/cc/v14 n1/v14n1a16.pdf

6 Ziliotto GC, Marcolan JF. Percepção de trabalhadores de enfermagem sobre sexualidade de portadores de transtornos mentais. Acta paul enferm (online) [Internet]. $2013 \mathrm{mai} / \mathrm{fev}$ [acesso em 2018 set 20];26(1):86-92. Disponível em: http://www.scielo.br/scielo.php?scrip $\mathrm{t}=\mathrm{sci}$ arttext\&pid=S0103-

21002013000100014\&lng=en\&nrm=iso \&tlng=pt

7 Ziliotto GC, Marcolan JF. Representações sociais da enfermagem: a sexualidade de portadores de transtornos mentais. REME rev min enferm [Internet]. 2014 jan/nov [acesso em 2018 set 20];18(4):966-72. Disponível em: http://www.reme.org.br/artigo/detal hes/976

8. Miranda FAN, Furegato ARF, Azevedo DM. Práticas discursivas e o silenciamento do doente mental: sexualidade negada?. Esc anna nery rev. Enferm [Internet]. 2008 mar [acesso em 2018 set 20];12(1):136-42. Disponível em: http://www.scielo.br/scielo.php?scrip $\mathrm{t}=\mathrm{sci}$ _arttext\&pid=S141481452008000100021

9 Soares NA, Silveira BV, Reinaldo MAS. Oficinas de sexualidade em saúde mental: relato de experiencia. Cogitare enferm [Internet]. 2010 abri/jun [acesso em 2018 set 20];15(2):345-8. Disponível em: https://revistas.ufpr.br/cogitare/arti cle/view/17874/11664

10 Barbosa JAG, Guimarães MDC, Freitas MIF. Sexualidade e vulnerabilidade social em face das infecções sexualmente transmissíveis em pessoas com transtorno mental. Rev méd Minas Gerais. [Internet]. 2013 out/dez [acesso em 2018 set 20];23(4):455-61. Disponível em: http://rmmg.org/artigo/detalhes/407

11 Pinheiro AS, Silva LRG, Tourinho, MBAC. A estratégia saúde da família e a escola na educação sexual: uma perspectiva de intersetorialidade [Internet]. Trab educ saúde. 2017 set/dez [acesso em 2018 set 20];15(3):803-22. Disponível em: http://www.scielo.br/scielo.php?scrip $\mathrm{t}=\mathrm{sci}$ arttext\&pid=S1981-

$77462017000300803 \& \operatorname{lng}=e n \& n r m=i s o$ \&tlng=pt

12 Hamann C, Pizzinato A, Weber JLA, Rocha KB. Narrativas sobre risco e culpa entre usuários e usuárias de um serviço especializado em infecções por HIV: implicações para o cuidado em saúde sexual. Saúde soc [Internet]. $2017 \mathrm{jul} / \mathrm{set}$ [acesso em $2018 \mathrm{set}$ 


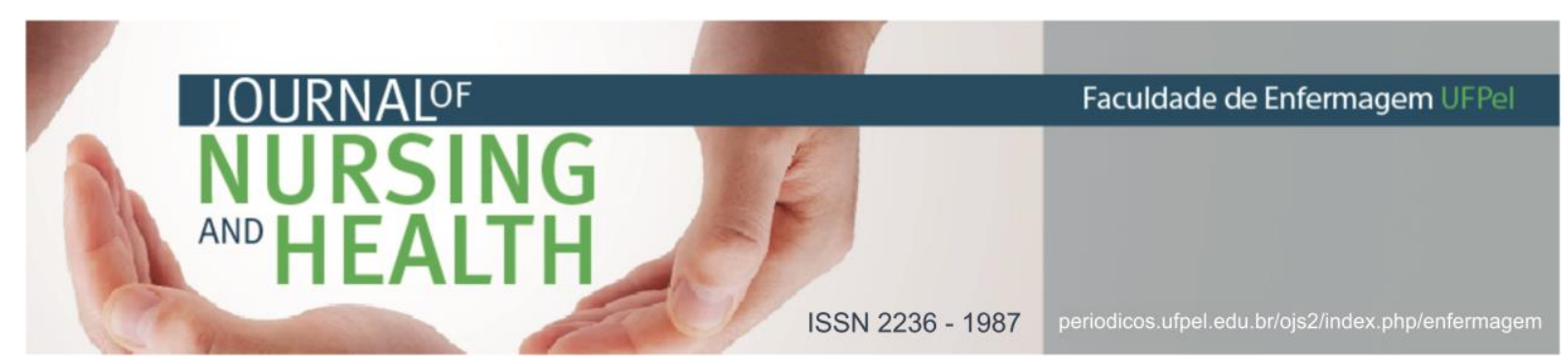

20];26(3):651-63. Disponível em: http://www.scielo.br/pdf/sausoc/v26 n3/0104-1290-sausoc-26-03-00651.pdf

13 Góis ARS, Oliveira DC, Costa SFG, Oliveira RC, Abrão FMS. Representações sociais de profissionais da saúde sobre as pessoas vivendo com HIV/AIDS. Av enferm [Internet]. 2017 mai/ago [acesso em 2018 set 20];35(2):171-80. Disponível em: http:// www.scielo.org.co/pdf/aven/v 35n2/0121-4500-aven-35-02-00171.pdf

Data de submissão: 30/04/2017

Data de aceite: 18/05/2018

Data de publicação: 29/10/2018 